on their way-living semi-pelagically-to the eastward, until the metamorphosis is completed, and the small eel young have acquired a dark covering of pigment. In Northern Europe they move-by way of the Channel and round the north of Scotland - through into the North Sea and farther, via the Danish waters, to the western parts of the Baltic, where they have been found so far east as E. of Bornholm, at stages where the metamorphosis was not yet quite completed (Dr. A. C. Johansen, with the Thor), Fig. x. In the northern parts of the Baltic, elvers are not known, or indeed any eels less than $20-30 \mathrm{~cm}$., though the eel occurs right in to the innermost waters of that sea. The eels found in Finland are large females, and on the east coast of Sweden no males have been found north of lat. $57^{\circ} \circ 8^{\prime}$ (off Öland); see Fig. I. This peculiar fact evidently answers to what we know from the great rivers, where the female eels generally move farther up into the higher reaches than the males.

The eel fry enter the Mediterranean at an early stage, as unmetamorphosed larvæ, most often not even having attained their full larval size, between one and a half and two years old. As unmetamorphosed larvæ they are found throughout the western basin, west of Italy, and at times, perhaps, still farther to the eastward. Even in the most easterly parts of the Mediterranean, an ascent of elvers takes place, these being transparent, and thus not having fully completed their metamorphosis. I have in this connexion received some information, with samples, from Mr. Geoffrey W. Paget, Director of Fisheries Investigations in Cairo. At a pumping station near Alexandria, where fresh water is pumped in large quantities into a channel leading direct to the sea, Mr. Paget found, on February 24, 1920, "that elvers were present in prodigious quantities, being unable to proceed further on account of the station. From this date-February 24-until April r $_{5}$, fishing was practically continuous, and we transported over 5,000,000 elvers to the canal systems inland." Mr. Paget's observations are highly interesting, showing as they do that elvers which have not yet completed their metamorphosis can occur in such great quantities so far east as about $30^{\circ} \mathrm{E}$. long. Together with observations from northern Europe, they give us a clear picture of the remarkable power of migration possessed by the eel fry. From the breeding grounds in the western Atlantic to the mouth of the Nile is a distance approaching 90 degrees of longitude, or one-fourth of the earth's circumference, and this distance is covered by the eel fry in the space of about three years. They may reach the Nile and the western Baltic before their metamorphosis is yet complete, and the greater part of the journey is made while they are still in the leaf-shaped larval stage. No other instance is known among fishes of a species requiring a quarter of the circumference of the globe to complete its life history, and larval migrations of such extent and duration as those of the eel are altogether unique in the animal kingdom.

\title{
Theories of Magnetism.
}

\section{By Dr. A. E. Oxuey.}

$\mathrm{M}$ $\mathrm{UCH}$ attention has been devoted in recent years to theories of magnetism, and an interesting survey of the position of the subject is given in the report of a committee of the U.S. National Research Council issued by the National Academy of Sciences, Washington, in August last (vol. 3, part 3). It is difficult in a descriptive article of moderate length to present judicially the various views which have been advanced, but an attempt will here be made to do this, using the report referred to as a basis, and supplementing it with accounts of one or two advances not recorded therein.

Poisson in 1820 published a mathematical theory of magnetism which was based on Coulomb's inverse square law. He merely regarded magnetic substances as possessing positive and negative magnetic fluids which could be separated by the application of an external magnetic field resulting in the production of the magnetic effects as observed in bar magnets. This theory was eventually (I83I) shown to be untenable by Faraday's discovery of the phenomenon of diamagnetism.

Ampère's theory (I825), based on Oersted's discovery (1820) of the magnetic effects of an electric current, may be regarded as the foundation of modern magnetic theories, though at that time the laws of electromagnetic induction were unknown. This theory led Weber (1854) to develop a theory which aimed at an explanation of the magnetic effects of bar magnets on the assumption that the molecules were always equivalent to miniature magnets, whether the substance were magnetised or not, the action of the external field being merely to align the miniature magnets along the direction of the applied field. No explanation of the phenomenon of hysteresis was given, however, until Maxwell ("Electricity and Magnetism," $\S$ 444) extended Weber's views and interpreted the more complicated hysteresis effects in terms of certain quasi-elastic forces.

The theory of Ewing ( 1890 ) enabled us to visualise the nature of these hypothetical controlling forces by attributing the sluggishness of the response to an applied field as due to the interaction between special groups of molecules. This gave a rough explanation of hysteresis effects in terms of the mutual actions between complex groups of molecular magnets, and accounted for the shape of the hysteresis loops, the coercive force and the retentivity of a ferro-magnetic substance like iron.

At the beginning of the present century, attempts were made by Voigt and J.J. Thomson to outline an electron theory of magnetism based on the magnetic effects of a moving electron, but it was not until the theory of paramagnetism and diamagnetism of Langevin appeared (I905) that a satisfactory interpretation of these phenomena was presented.

The classical researches of Curie (1895) had shown that substances could be divided into three groups as regards their magnetic properties under an external field. These are, (I) diamagnetic substances, which show a minute negative induced moment, practically independent of temperature; (2) paramagnetic sub- 
stances, which show generally a larger and positive induced moment, varying inversely as the absolute temperature, and (3) ferro-magnetic substances, which show still larger positive magnetic moments, which vary with the temperature and external field in a complex way. In each case the total induced moment per gram of the substance, divided by the applied field, is called the specific susceptibility. On Langevin's theory, a molecule consists of a congeries of revolving positive and negative charges; if the total initial magnetic moment of these is zero, the substance is diamagnetic, if it is not zero, the substance is either paramagnetic or ferro-magnetic. The diamagnetic effect must exist in all matter, but is masked by the larger para- or ferro-magnetic effects in the latter case. Langevin's theory indicated that when there is no interaction between the molecules, the diamagnetic effect is independent of temperature, while the paramagnetic effect varies inversely as the absolute temperature in accordance with the Curie rules mentioned above. Langevin did not consider ferro-magnetism; this was done by Weiss.

These remarks hold only in so far as there is no appreciable mutual action between the molecules. In ferro-magnetic substances, such action is pronounced, and Weiss (I907) extended Langevin's theory by introducing an intrinsic molecular field to represent this mutual molecular interference. According to Weiss the molecular field has not necessarily a magnetic origin; it corresponds to the forces determining crystallisation, but for magnetic purposes it may be regarded as a magnetic field proportional to the intensity of magnetisation, and its value is then of the order $10^{7}$ gauss. Weiss further showed that the energy of this field is a measure of the thermal change when, at the critical temperature, the substance passes from the ferro-magnetic to the paramagnetic state. The results obtained with magnetite above the critical temperature showed that Curie's rule of paramagnetism held but that the constant of proportionality had a series of different values over certain temperature ranges. These values were interpreted by Weiss as due to sudden changes of the molecular magnetic moment by a unit, the value of which was found to be $16.4 \times 10^{-22}$ c.g.s.e.m.u. This is the Weiss magneton; its value has later been corrected to $18.54 \times 10^{-22}$. Weiss and others claim that this unit exists in many ferro-magnetic substances and in paramagnetic salts, though in the latter substances the evidence is not quite so conclusive. Further practical and theoretical extensions of the work have been made by Weiss, Kunz, Honda, and Frivold, but lack of space prevents an extended account of these here.

Honda (rgro) made an extensive examination of the variation of susceptibility of many elements with temperature, and concluded that, in general, the Curie rules did not hold. In I9I4 he submitted that the magnetic moments of molecules were not constant but depended on the temperature, and that they exert forces on one another which hinder their lining up parallel to the field. In solids which are paramagnetic, the magnetic unit is a spherical group of molecules. This sphere becomes elongated in the ferromagnetic state. A second theory due to Honda (I9I4) advocates a gyroscopic motion of the molecule to account for diamagnetism and paramagnetism. This is very similar to the theories of Gans (I9I0-I9I6). There appears to be no doubt that certain gyroscopic motions are involved, but more recent evidence (see below) indicates that these do not arise from molecular rotations but from a gyroscopic property of the electron itself, i.e., the electron is a magneton.

Certain departures from the Curie rules for paramagnetic crystals at low temperatures have been examined by Onnes, Oosterhuis, and others, and interpreted in terms of a molecular field in a manner similar to that of Weiss.

The variation of diamagnetism accompanying the transition from the liquid to the crystalline state has been investigated by the writer (1911-22), who found that organic compounds changed their specific susceptibility by a few per cent. The theoretical explanation of the results was obtained by including in the Langevin theory of diamagnetism a term depending on the local polarisation which determines a local molecular field. Weiss regarded his molecular field as uniform, but in the present case it must be of an alternating character as we pass from molecule to molecule of the crystal. It exists whether the substance is subjected to an external field or not, and distorts the electron orbits, producing a few per cent. change of specific susceptibility on crystallisation. It can be shown that (r) these local fields are of the same order of intensity as Weiss's field, namely, Io ${ }^{7}$ gauss, (2) the energy of this field is a measure of the latent heat of fusion, (3) the existence of such a field would induce a magnetic double refraction which is comparable with the natural double refraction of crystals, (4) the change of volume on crystallisation is the magneto-striction effect of this molecular field, and (5) the energy of the local molecular field per unit volume represents the tensile strength of the crystal.

Thus it appears that in all crystalline media there are intense local fields, the linking up of which from molecule to molecule determines the rigidity of the crystal. We are not certain what is the true nature of the field ; it is probably partly electrostatic and partly magnetic. That the magnetic forces are important in determining the distribution of the planes of cleavage in crystals has been emphasised by the writer (1920), a uniform magnetic field being capable of isolating the cleavages, i.e., of distinguishing between an open or close packing of the molecules in certain directions.

The present position of magnetic theories is fascinating. There appears to be evidence that the ultimate magnetic particle is neither the molecule nor the atom but the electron itself ; in other words, the electron is not merely an electrostatic charge but also a magnetic doublet or magneton. Such a structure no doubt accounts for the spiral tracks of the $\beta$-particles as observed by C. T. R. Wilson. The problem of the interaction between such doublets in crystalline media is far from being solved. It appears that a useful picture of the mechanism is obtained on the LewisLangmuir theory (elaborated by Langmuir in 1919) of the cubical atom. In non-ionised media the coupling force between atoms is formed by units, each consisting of a pair of electrons, and each pair corresponds to a single valency bond of chemistry. The influence of 
magnetic forces in determining crystalline structure, magne-crystallic action, and chemical combination in non-ionised media is apparent. It is interesting to note that Pascal (19ro) showed that in organic compounds, all of which are diamagnetic, the molecular susceptibility is (apart from certain peculiarities of structure common to certain types of compounds) equal to the sum of the atomic susceptibilities of the component atoms. This is not true of ionised compounds, such as metallic salts, where the coupling between the atoms is probably of an electrostatic nature.

Further developments of the magneton theory were made by Parson (I9I5), who identified the electromagnetic coupling between pairs of magnetic doublets with the force of chemical combination. The magneton, or anchor ring electron, has been applied by Allen (1920) to interpret the phenomena of optical activity and optical isomerism. In connexion with the magnitude of the local magnetic field, namely, $10^{7}$ gauss, it is interesting to note that Allen's calculations give a value $10^{8}$ gauss at a distance from the anchor ring equal to its radius.

A number of attempts to obtain a quantum theory of magnetism have been made in recent years by Oosterhuis, Keesom, Gans, Reiche, and others. These are based on the assumption that the molecules are endowed with quantised molecular rotations, but the theory of Gans is the only one to take account of molecular interactions.

In connexion with these views the theory of Bohr and Sommerfeld must be considered. Though this has proved so successful in the interpretation of the fine structure of spectral lines, it does not appear at all obvious how the open elliptical orbits of this theory can give the uniquely balanced systems required to explain diamagnetism, nor does it give a picture of the directed forces which are responsible for crystal lattices. These considerations suggest that the atom must have a static structure. Perhaps the electron itself is quantised, the motion of its parts being highly localised compared with atomic dimensions. The electrons in an atom may be distributed on spherical or ellipsoidal surfaces, and the passage from one surface to another determine the emission of a definite amount of radiation of a certain frequency.

Quite recently Whittaker (1922) has published a new quantum mechanism of the atom based upon the existence of a number of atomic magnetic doublets. If an electron collides with this system the collision is perfectly elastic if the velocity of the electron is less than a certain amount. If the velocity exceeds this amount the electron passes through the magnetic system and hands over to the latter a definite quantum of energy which is identified as Planck's quantum. The derivation of the Balmer series can be obtained from this conception; it may later be found equally effective in interpreting the fine structure of spectral lines. Allen has replaced the particular magnetic structure postulated by Whittaker by a pair of ring electrons, thus identifying Whittaker's model more closely with Langmuir's cubical atom. The atomic structure is dynamical locally but is essentially static at ranges comparable with molecular dimensions. The static structure is required to account for crystalline and magnetic properties of matter in the non-radiating state. Recent experiments by the writer (1922) indicate that the occlusion of hydrogen by palladium produces a system the electronic configuration of which is similar to that of silver, and the fall in paramagnetism of the palladium is consistent with this view, silver being diamagnetic. Manganese which has been fused in an atmosphere of hydrogen is ferro-magnetic, although pure manganese is paramagnetic. Iron which has been fused in hydrogen has a higher coercive force than ordinary iron (like cobalt). These experiments indicate that when hydrogen is occluded in one of these elements an electronic system is produced corresponding to an element the atomic number of which is one higher than that of the element occluding the hydrogen. The suggestion is that the hydrogen electron, in such systems, enters into the outer shell of electrons of the metallic atom.

A static model, consistent of course with a highly localised dynamical model, such as the one advocated above, seems to be the only satisfactory interpretation of these results.

\section{Obituary.}

\section{Prof. Oscar Hertwig.}

$\mathrm{T}$ E death of Oscar Hertwig, formerly professor of anatomy in the University of Berlin and director of its Anatomical-Biological Institute, removes from the scene one of the chief leaders in morphological science. He formed a link in that chain of illustrious men including Johannes Müller, Gegenbaur, Fürbringer, and Gaupp, which has demonstrated how fully Germany has realised the importance of entrusting its great chairs of anatomy to men who are anatomists in the broadest sense of the word, leaders in vertebrate morphology and not merely experts in the details of anthropotomy.

Hertwig was most widely known through his series of admirable text-books. His "Lehrbuch der Entwicklungsgeschichte des Menschen und der Wirbeltiere " made its appearance in $\mathbf{I} 886$ and has passed through numerous editions, both in its extended and in its condensed form ("Elemente," 3 rd Edition, I920). "Die Zelle und die Gewebe," first published in 1893 , and known in its later editions as the "Allgemeine Biologie," is still widely used as a most admirable text-book of general biology on a cytological basis. During the years r9or-6 Hertwig brought out the various instalments of that wonderful encyclopædia which bears the characteristically German title "Handbuch der Entwicklungslehre der Wirbeltiere," edited and in parts written by himself. While it is, perhaps, permissible to hope that the appearance of this colossal work marks the approaching end of what may be called the encyclopædic age of biology, in which real progress has become more and more impeded and slowed down by the accumulation of minute details, there can be no question regard- 